\title{
Probing phenotypic growth in expanding Bacillus subtilis biofilms
}

\author{
Authors: Xiaoling Wang, Stephan A. Koehler, James \\ N. Wilking, Naveen N. Sinha, Matthew T. Cabeen, \\ Siddarth Srinivasan, Agnesen Seminara, Qingping \\ Sun, Michael P. Brenner, \& David A. Weitz.
}

The final publication is available at Springer via https://doi.org/10.1007/s00253-016-7461-4.

Wang, X., Koehler, S. A., Wilking, J. N., Sinha, N. N., Cabeen, M. T., Srinivasan, S., ... Weitz, D. A. (2016). Probing phenotypic growth in expanding Bacillus subtilis biofilms. Applied Microbiology and Biotechnology, 100(10), 4607-4615. doi:10.1007/s00253-016-7461-4 


\section{Probing phenotypic growth in expanding Bacillus subtilis biofilms}

Xiaoling Wang, Stephan A. Koehler, James N. Wilking, Naveen N. Sinha, Matthew T. Cabeen, Siddarth Srinivasan, Agnese Seminara, Shmuel Rubinstein, Qingping Sun, Michael P. Brenner, \& David A. Weitz

We develop an optical imaging technique for spatially and temporally tracking biofilm growth and the distri-bution of the main phenotypes of a Bacillus subtilis strain with a triple-fluorescent reporter for motility, matrix production, and sporulation. We develop a calibration procedure for deter-mining the biofilm thickness from the transmission images, which is based on BeerLambert's law and involves cross-sectioning of biofilms. To obtain the phenotype distribution, we assume a linear relationship between the number of cells and their fluorescence and determine the best combination of calibration coefficients that matches the total number of cellsfor all three phenotypes and with the total number of cells from the transmission images. Based on this analysis, we re-solve the composition of the biofilm in terms of motile, ma-trix-producing, sporulating cells and low-fluorescent materials which includes matrix and cells that are dead or have low fluorescent gene expression. We take advantage of the circular growth to make kymograph plots of all three

phenotypes and the dominant phenotype in terms of radial distance and time. To visualize the nonlocal character of biofilm growth, we also make kymographs using the local colonization time.

Our tech-nique is suitable for real-time, noninvasive, quantitative stud-ies of the growth and phenotype distribution of biofilms which are either exposed to different conditions such as biocides, nutrient depletion, dehydration, or waste accumulation.

Keywords Bacillus subtilis . Phenotype visualization . Growth characterization . Biofilm . Phenotype switching

\section{Introduction}

Biofilms are communities of tightly associated bacteria encased in an extracellular matrix and attached to liquid or solid surfaces (Vlamakis et al. 2013). In medical settings, their growth can lead to numerous problems, such as infections (Costerton et al. 1999; Hall-Stoodley and Stoodley 2009), whereas their growth can be desirable in agricultural and in-dustrial settings such as wastewater treatment, bioremediation (Singh et al. 2006; Eisele and Gabby 2014), and microbial fuel cells (Gardel et al. 2012; Logan 2008). The ability of bacteria to differentiate into multiple phenotypes is crucial to biofilm growth, as it enables adaptation to environments and hence bacterial survival in unfavorable conditions, such as high temperature and pressure, extreme $\mathrm{pH}$, intense $\mathrm{UV}$ radiation, or 
limited nutrient availability (Hottes et al. 2005; Kearns et al. 2005)

Bacillus subtilis is one of the most-studied Gram-positive bacteria and is considered a good model organism to study biofilm formation (Golden and Yoon 2003). Its uses include treatments for gastric and urinary tract diseases (Doherty et al. 2010), improving agricultural yields and being produced to be a Japanese delicacy known as "natto" (Swain and Ray 2009). Its main phenotypes during biofilm growth are (i) cells that are motile, (ii) cells that produce extracellular matrix, and (iii) cells that differentiate into spores. Motile cells have flagella that enable them to swim in solution or to swarm along wet surfaces (Kearns and Losick 2005; Branda et al. 2001). Matrix-producing cells produce a mixture of amyloid proteins and exopolysaccharides that hold the community together (Vlamakis et al. 2008). Spores are metabolically inactive, resistant to heat and antibiotics, and can germinate under favorable conditions (Rudner and Losick 2001; Piggot and Hilbert 2004). These three phenotypes are mutually exclusive, and together, they account for nearly all of the cells (Curtis et al. 2007). Additional cell states include competent cells, which are able to take up extracellular DNA from their environment (Dubnau 1991; Dubnau and Provvedi 2000), and cannibalistic cells, which can kill their sister cells by secreting toxins or and utilize them as a nutrient source (Gonzalez-Pastor et al. 2003). Thanks to phenotypic differentiation, the bacterial community can optimize its resources and become resistant to harsh environmental conditions.

Although many details of how the genetic circuits controlling these different cell states are understood at the single-cell level (Vlamakis et al. 2008), organization of bacterial colonies on the mesoscopic level remains poorly understood. This is due in part to limited availability of experimental techniques for observing the changes in genetic state of bacteria throughout space and time during the growth of the colony. Existing techniques for monitoring phenotypes in growing biofilms include flow cytometry and fluorescent microscopy using bacterial strains with fluorescent reporters for one or two phenotypes (Mazza 1994; Evdokimov et al. 2006; Vlamakis et al. 2008; Falk et al. 2009; Chudakov et al. 2010; Verplaetse et al. 2015; Seminara et al. 2012). Flow cytometry removes specific regions of the biofilm and then disperses individual cells in solution for analysis, which destroys the biofilm. Therefore, obtaining detailed spatial distributions of cell state over time with flow cytometry is impractical, as every time point requires the coordinated removal and analysis of many small biofilm pieces. Fluorescence microscopy of biofilms with fluorescent phenotype reporters is a nondestructive method with high resolution and therefore is a promising alternative technique. However, currently only one or two cell types have been fluorescently tagged at a time (Vlamakis et al. 2008; O'Toole et al. 2000; Chang and Cohen 1979), which is insufficient for fully understanding biofilm evolution. Moreover, previous fluorescent biofilm analyses (Vlamakis et al. 2008) were performed using serial sectioning, thus again destroying the biofilm and not giving a full spatial time course of its development. Accordingly, a nondestructive technique for spatiotemporal quantification of the main phenotypes of a growing biofilm remains lacking.

Here, we develop a nondestructive method for the analysis of bacterial biofilm growth based on optical transmission and fluorescence microscopy using a triple-labeled B. subtilis strain. This method reveals the spatial and temporal distribution of cell states, as well as that of low-fluorescent or nonfluorescent materials, such as the extracellular matrix or cells that are either dead or have decreased fluorescent expression levels.

\section{Materials and methods}

We grew colonies on $1.5 \mathrm{wt} \%$ agar gel containing minimal salts glutamate glycerol (MSgg) medium designed to induce biofilm formation and sporulation. MSgg is composed of $5 \mathrm{mM}$ potassium phosphate ( $\mathrm{pH} 7), 100 \mathrm{mM}$ MOPS ( $\mathrm{pH} 7)$, $2 \mathrm{mM} \mathrm{MgCl}_{2}, 700 \mu \mathrm{M} \mathrm{CaCl}, 50 \mu \mathrm{M} \mathrm{MnCl}_{2}, 50 \mu \mathrm{M} \mathrm{FeCl}_{2}$, $1 \mu \mathrm{M} \mathrm{ZnCl}_{2}, 2 \mu \mathrm{M}$ thiamine, $0.5 \%$ glycerol, $0.5 \%$ glutamate, $50 \mu \mathrm{g} / \mathrm{ml}$ tryptophan, $50 \mu \mathrm{g} / \mathrm{ml}$ phenylalanine, and $50 \mu \mathrm{g} / \mathrm{ml}$ threonine. The agar solution is cooled to $55^{\circ} \mathrm{C}$ before adding the remaining ingredients. We use $10-\mathrm{cm}$-diameter petri dishes containing a 2-mm-thick layer of agar substrate; we cover the dishes with lids, let them cool overnight at room temperature, and spot within $24 \mathrm{~h}$. These conditions result in bacterial colonies that reproducibly exhibiting the main phenotypes within a span of 2 days.

We constructed a triple-reporter B. subtilis strain (3610 sacA::Phag-mkate $2\left[\mathrm{Kan}^{\mathrm{R}}\right]$ amyE::P tap $A-c f p\left[\mathrm{Spc}^{\mathrm{R}}\right]$ ywrK::P $P_{\text {ssp }}$-citrus $\left.\left[\mathrm{Cm}^{\mathrm{R}}\right]\right)$ to visualize different cellular differentiation states. This strain, MTC871 (available from the Losick laboratory), is based on the biofilm-forming NCIB3610 "wild" strain (RL2912 in the Losick laboratory). The red fluorescent protein mKate 2 reports on the hag promoter, which is responsible for flagellin production in motile cells. The blue fluorescent protein CFP reports on the tapA promoter, which corresponds to the amyloid protein in matrixproducing cells. A yellow fluorescent protein, citrus, reports on the $\operatorname{ssp} B$ promoter, which is expressed at a late stage in sporulating cells. When a particular promoter for a phenotype is expressed, the corresponding fluorescent protein is also produced. There is no spectral overlap of the three fluorophores, except some minor agar autofluorescence in the CFP channel. Moreover, accumulation of pigment becomes noticeable after 1 day and diminishes transmitted light, but as our experiments are limited to 2 days, we neglect the effects of the pigment. 
We transferred the bacteria to the surface of the agar by spotting with $0.1 \mu \mathrm{l}$ of bacterial culture at $\mathrm{OD}_{600}=1$. Before inoculating the plates, we removed the lids and allowed the surface to dry for 5-10 $\mathrm{min}$. We allowed the drop to dry for 510 additional minutes with the lid off, until the meniscus of the initial drop was no longer visible and the bacteria were left in a "coffee ring" around the perimeter whose radius is about $1 \mathrm{~mm}$. To prevent evaporation during recording of timelapse movies, we placed the spotted plate in a Tupperware container stuffed with wet paper towels and sealed around the microscope using Glad Press'n'Seal plastic wrap. The temperature of the microscope was maintained at $32{ }^{\circ} \mathrm{C}$ using heating elements and fans.

The biofilm produces a pigment over time, which changes the attenuation of the white light through the colony material over time. The function of this pigment is poorly understood (Shemesh and Chai 2013), but it appears to be nonenzymatically produced water-soluble molecules associated with the intercellular pH (Barnett et al. 1983). To compensate for this effect, we repeat the experiment with colonies of different ages. This pigment does not absorb infrared light (IR), so we compare images with and without an IR filter in the transmitted light path to isolate the effect of the pigment, as shown in Fig. 1.

\section{Results}

We made time-lapse movies of the growing biofilm by recording the three fluorescent channels and the transmitted light channel. After about $10 \mathrm{~h}$, the biofilm becomes visible to the naked eye and it continues to grow in a circular fashion such that after $58 \mathrm{~h}$, its diameter is $20 \mathrm{~mm}$, as shown in Fig. 2 and Movie S1 (A). The images show that the different phenotypes are spatially and temporally organized. cells begin to sporulate after $30 \mathrm{~h}$; however, over the duration of the experiment, sporulating cells are not present in the center of the biofilm. In contrast, motile cells are predominantly located in the middle of the biofilm. Matrix-producing cells are present throughout, but they predominate at the outer ring of the biofilm. We performed a detailed analysis of these movies to provide a quantitative description of biofilm growth.

To estimate the biofilm thickness from transmission images, we developed a calibration procedure involving crosssectioning of biofilms. For this, we used a constitutively fluorescent B. subtilis strain, NCIB 3610, which was obtained from the Kolter lab. We measured the optical density, defined as Eq. (1).

$O D=-\log _{10}\left(\frac{I}{I_{0}}\right)$

where $I$ is intensity of the transmitted light through the substrate and biofilm, and $I_{o}$ is that through the transparent substrate. Similar to previous studies (Mazza 1994; Dervaux et al. 2013; Wiedenmann et al. 2009), we use the Beer-Lambert law to estimate the biofilm thickness $(h)$ from the optical density (OD) which is shown in Eq. (2).

$O D=h / \lambda$

where $\lambda$ is the attenuation length. To determine $\lambda$, we compare the transmission with the height obtained from the side view of a sectioned biofilm formed from the constitutively labeled strain, which, compared with the triple-labeled strain, has
Fig. 1 Correction for pigment using IR filter, both images show a biofilm on an agar plate, half the colony has been scraped away to reveal the agar beneath it. a With the regular bandpass filter used for transmitted light images, the light intensity decays toward the center of the colony due to pigment produced by the colony. b With an IR filter in the transmitted light path, the transmitted light profile is more uniform

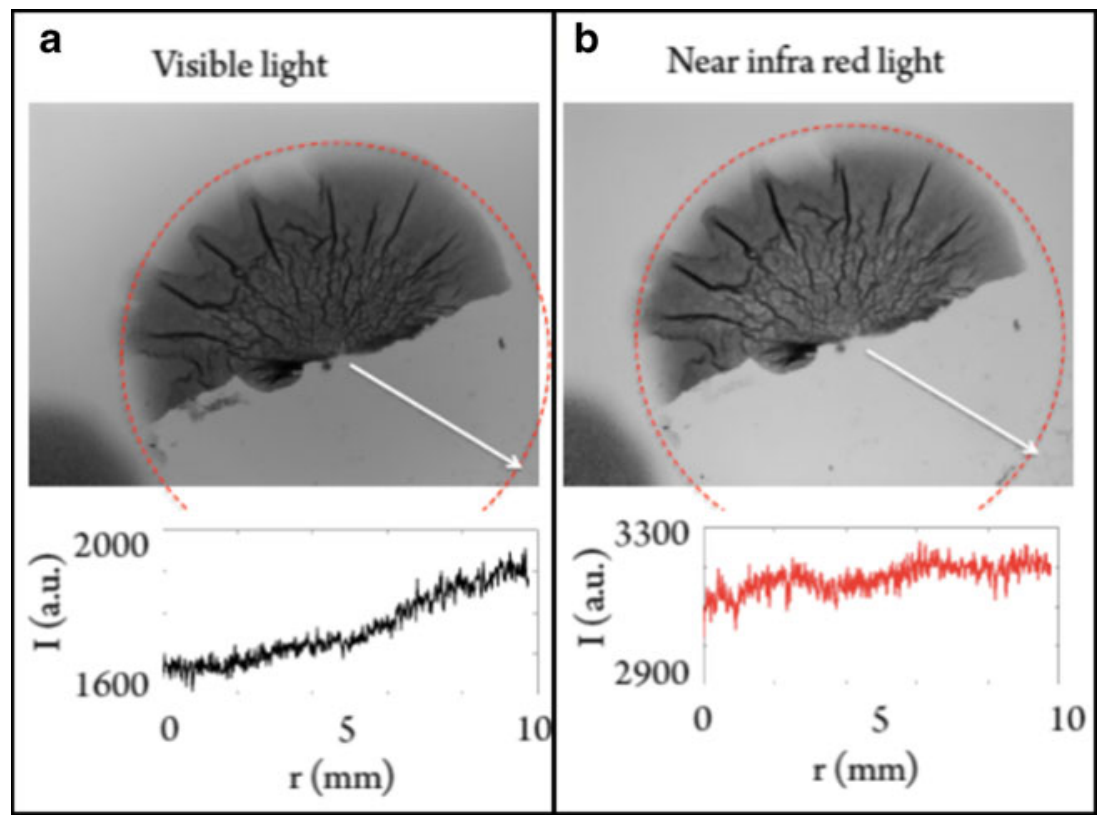




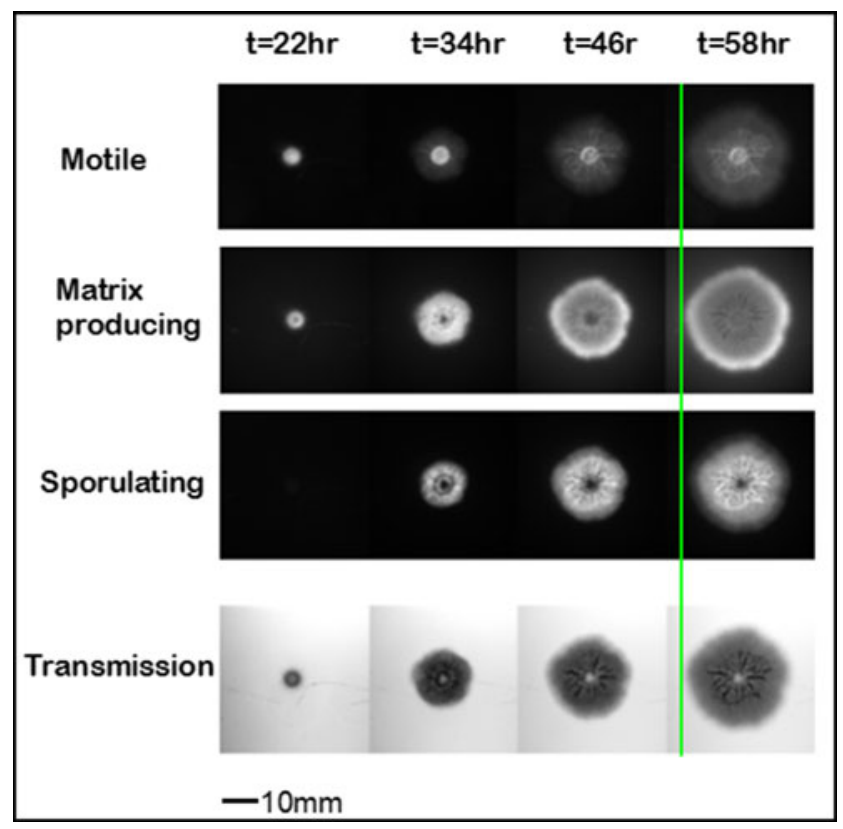

Fig. 2 Time-lapse images of a growing Bacillus subtilis biofilm at four different times after inoculation, namely $t=22,34,46$, and $58 \mathrm{~h}$. Upon excitation, the motile, matrix-producing and sporulating phenotypes produce different fluorescent colors and are observed using red, blue, and yellow filters, respectively. The transmission images are shown at the bottom and are used to determine the optical density. The green line serves as guide, indicating that at $58 \mathrm{~h}$, the extent of the matrix-producing cells is comparable to that of the motile cells and exceeds that of the sporulating cells (color figure online)

minimal spatial variation in the fluorescence signal. We reinforced the biofilm for subsequent manipulations by covering it with a layer of agar. We cut a thin slab of this biofilm-agar sandwich, whose top view is shown by the transmission image in Fig. 3a. The transmission, $I / I_{0}$, is determined from the ratio of the transmitted light relative to that of the region not containing biofilm, and we obtain the transmission trace by averaging along the transverse direction. We next obtain the biofilm height by flipping the slab onto its side and imaging its cross section using the constitutive fluorescent channel, as shown in Fig. 3b. We then register the transmission and height traces to obtain the linear relationship given by Eq. (2), thereby determining the attenuation length, $\lambda \sim 1.2 \mathrm{~mm}$, as shown in Fig. 3c. The attenuation length appears to change with biofilm age, which we attribute to darkening resulting from the accumulated pigment. The correlation coefficient of the linear fit is $R^{2}=0.7$, and we estimate the uncertainty of the estimated biofilm thickness to be about $30 \%$.

We characterized the biofilm growth based upon the height profiles obtained from the optical density. The biofilm colony shows dramatic ridges whose height approaches $0.5 \mathrm{~mm}$, whereas the average height approaches $0.2 \mathrm{~mm}$, as shown in Fig. 4a. Our transmission measurements are able to detect heights greater than $15 \mu \mathrm{m}$, which we used as a threshold to discriminate regions colonized by the biofilm from uncolonized regions. Using this threshold, we obtain the average colony widths along all axes. This average width displayed a constant increase for about 1 day, with an expansion rate of $0.31 \mathrm{~mm} / \mathrm{h}$, whereas the rate slows down at later times (Fig. 4b). The biofilm volume initially increases rapidly with time, but it increases linearly after 1 day, as shown in Fig. 4c.

We next determined the evolution of the three main phenotypes by analyzing the pixel intensities of the fluorescence movies, where each pixel images a vertical projection of a column of biofilm of height $h$. Applying arguments similar to those used in Beer-Lambert's law, the measured fluorescence is shown in Eq. (3).

$F=\alpha I_{\text {excitation }} \int_{0}^{h} e^{-z / \lambda^{*}} \rho(z) d z / \lambda^{*}$

where $\lambda^{*}$ is the effective attenuation coefficient of both the excitation and fluorescent light, $\alpha$ is a conversion efficiency, $I_{\text {excitation }}$ is the excitation light intensity, and $\rho$ is the concentration of fluorophores. We make the simplifying assumptions that the fluorescence of each cell is either fully "on," $F^{\text {on }}$, or completely "off," and that the biofilm is thin enough that the attenuation of both excitation and fluorescence light is negligible. This leads to a linear relationship between the measured
Fig. 3 Calibration procedure for determining biofilm thickness from the transmission images. a Top view of the biofilm slice using false coloring for the transmission. b Side view of the biofilm slice using the constitutive fluorescent channel. c The calibration curve is obtained from the relationship between the biofilm optical density and thickness

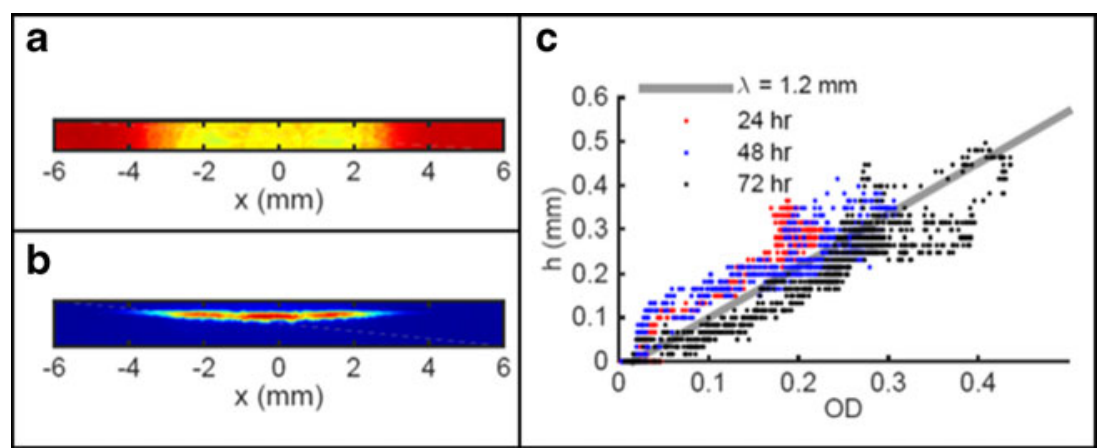


a

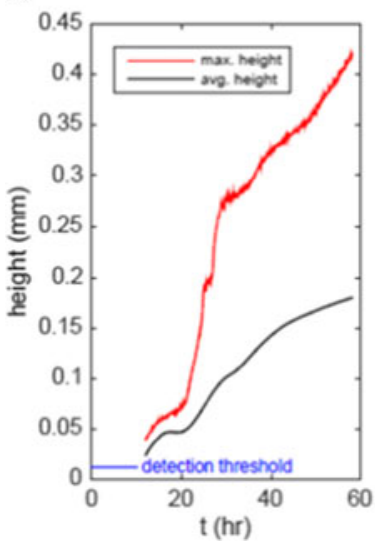

b

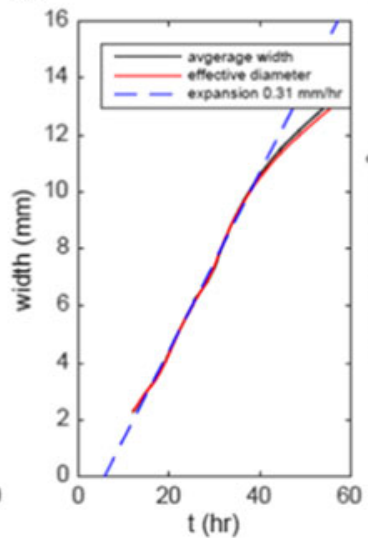

C

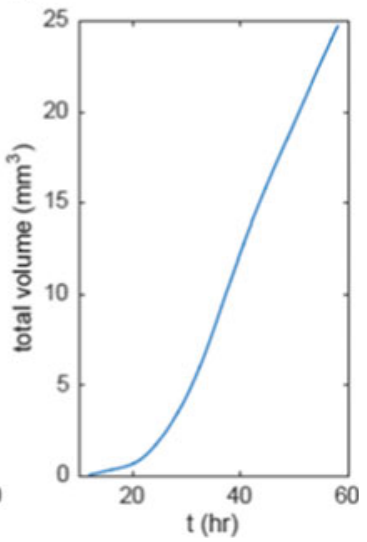

Fig. 4 Biofilm growth over $60 \mathrm{~h}$. a The maximum height and average height, which is the ratio of the volume and the in-plane area. $\mathbf{b}$ The average width of the biofilm, which is the mean diameter taken along all directions, and the effective diameter, which is obtained from the surface area projected in the plane, $D=2 \sqrt{A / \pi}$. The expansion rate for the first day is $0.31 \mathrm{~mm} / \mathrm{h}$, as indicated by the dashed line. $\mathbf{c}$ The volume increases quadratically with time at the beginning, and thereafter increases linearly fluorescence and the number of fluorescing cells within the column, which is shown as Eq. (4).

$F_{i}=n_{i} F_{i}^{\text {on }}$

where $i$ denotes the three different states: motile, matrix-producing, and sporulating. Because cells are in only one of three possible states, the following relation holds at all times, which is shown in Eq. (5):

$n=n_{\text {motile }}+n_{\text {matrix }}+n_{\text {sporulaton }}$,

where $n$ is the total number of cells inside a vertical column imaged by a pixel. The total number of cells is $n=h \Phi / h_{\text {cell }}$, where $\Phi$ is the average volume fraction of cells in the biofilm and $h_{\text {cell }}$ is the average height of one cell. Accordingly, the local thickness $h$, determined by Beer-Lambert's law, Eq. (2), should be equivalent to a linear sum of the fluorescence, which is shown in Eq. (6).

$h=C_{\text {motile }} F_{\text {motile }}+C_{\text {matrix }} F_{\text {matrix }}+C_{\text {sporulating }} F_{\text {sporulating }}$

where $C_{i}=h_{\text {cell }} /\left(\Phi F_{\mathrm{i}}{ }^{\text {on }}\right)$. We expect this relationship to be inaccurate for early times because the detected fluorescence is too weak. Moreover at later times, when the biofilm is thicker, $100 \mu \mathrm{m} \leq h \leq 200 \mu \mathrm{m}$, light attenuation can result in underestimates of 30 to $50 \%$. More importantly, we expect that Eq. (6) leads to thickness underestimates, as the amount of nonfluorescing materials, such as matrix, dead, or dormant cells increases.

We determined the best set of fluorescence calibration coefficients over the time interval $0<t<T$ by assuming $\Phi \approx 1$ and solving the over-determined system of linear equations given by Eq. (6) for every pixel of the biofilm image. The optimal solution occurs when the relative error between the biofilm volume as measured from the transmission or with the combined fluorescence is minimal, which we expect to occur before the significant accumulation of nonflourescent and low-fluorescent material as gene expression starts to decrease, which occurs after $24 \mathrm{~h}$ (Vlamakis et al. 2008). Accordingly, the optimal time interval for calibrating the fluorescence coefficients is $T=35 \mathrm{~h}$, as shown in Fig. 5a. For longer times, $t>T$, the amount of material with low or no fluorescence becomes significant, the biofilm is thick enough such that light attenuation is nonnegligible, and thus, estimates based solely on fluorescence become increasingly worse, as shown by the diverging red curve in Fig. $5 \mathrm{~b}$. We take the difference between the heights obtained from the optical density and fluorescence measurements as estimates of the amount of low-fluorescence materials. We observe that after $35 \mathrm{~h}$, the volume of the lowfluorescence materials is proportional to that of sporulating cells, as shown in Fig. 5c. The proportionality factor of 1.8 indicates that for every sporulating cell there is twice that volume of low-fluorescing cells and matrix. This observation is qualitatively consistent with the nutrient depletion-driven connection between cell death and sporulation. However, at the limit of long times, as cells die due to insufficient nutrition, the expression of fluorescence genes inevitably stops, and we expect Eq. (6) to completely break down.

Based on the measured biofilm thickness and fluorescence, we can also determine the fractional composition of the biofilm colony over time by using the fluorescence calibration factors, as shown in Fig. 6 . For short times, $t<18 \mathrm{~h}$, the fluorescence signal is too weak for our method to accurately determine the composition. In Fig. 6, we include published flow cytometry measurements of four biofilms with different ages (Vlamakis et al. 2008), as shown by the bars. Our biofilms were grown on a 2 -mm-thick agar substrate in a warmed microscope chamber, whereas the biofilms for the flow cytometry study were grown on thicker agar substrates 


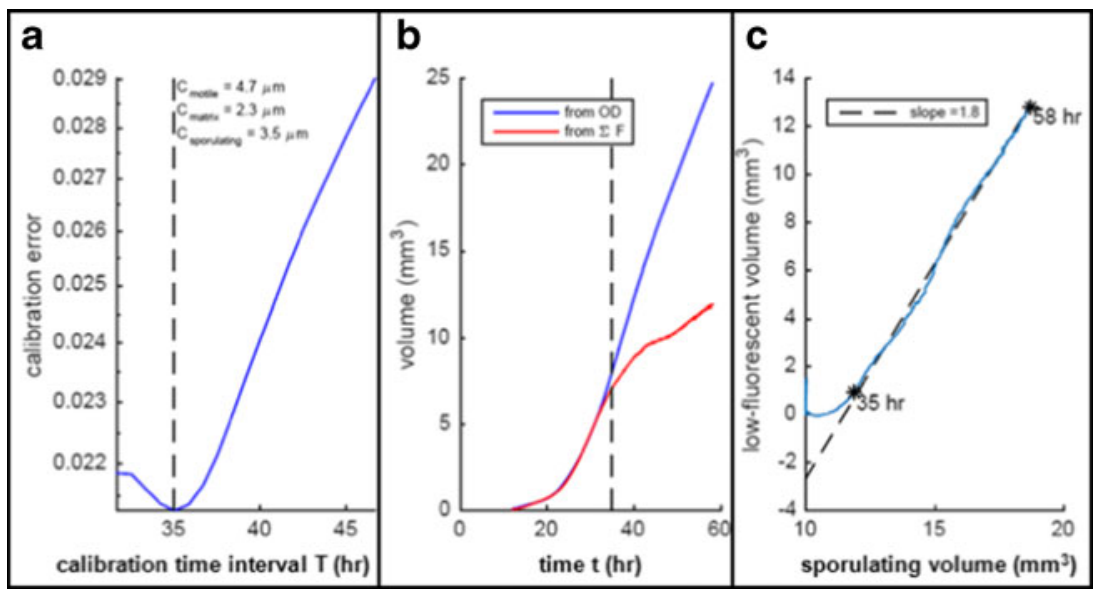

Fig. 5 a Dependence of the fluorescence calibration error on the time interval; at $35 \mathrm{~h}$, this error has a local minimum and starts to rise due to an increase of the low-fluorescence material. $\mathbf{b}$ The growing biofilm volume obtained from the transmission measurements and the fluorescence

in incubators at $30^{\circ} \mathrm{C}$. Considering the differences in growth conditions, the agreement is good and confirms our analysis. The fractional composition of the motile and sporulating phenotypes has $20 \%$ or better agreement, and the largest discrepancy is for the matrix-producing phenotype at $48 \mathrm{~h}$. However, given the large decrease in expression of the matrix gene after 1 day (Vlamakis et al. 2008), a massive underestimate is expected. According to our classification scheme, after 1 day, most matrix-producing cells are considered to be lowfluorescence materials. Movie S1 (E) shows the effective height of low-fluorescence material.

The radially symmetric growth conveniently lends itself to making kymographs, where the spatial dimension is the radius and azimuthal averages of the quantity of interest are taken. The height of the biofilm monotonically increases with time and is greatest for radial distances $0.5<r / \mathrm{mm}<2$, as shown in Fig. 7a. In Fig. 7b, we show the spatiotemporal dependence of

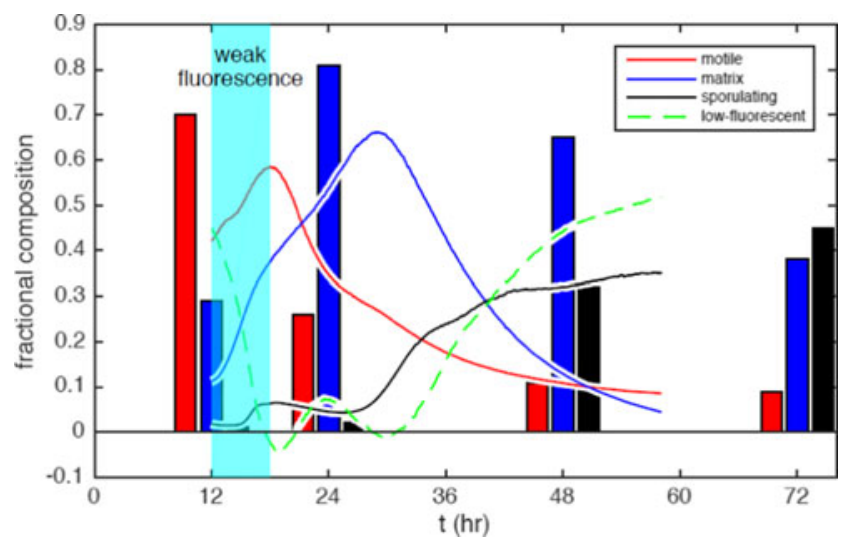

Fig. 6 Fractional composition of the different phenotypes over time, with curves overlaid on flow cytometry measurements taken at 12, 24, and $48 \mathrm{~h}$ (Doherty et al. 2010). Due to weak fluorescence, the estimated fractional composition at early times, $t<18 \mathrm{~h}$, is unreliable, as indicated by the shaded region signal, using the calibration constants in Fig. 4a. c The correlation between the low-fluorescence volume and the sporulating cell volume is linear between 35 and $58 \mathrm{~h}$; at earlier times, the low-fluorescence volume is negligible

the discrepancy between the height obtained from the transmission using Eq. (2) and that from the fluorescence channels using Eq. (6). For short times, $t<35 \mathrm{~h}$, the amount of lowfluorescence material is small and, aside from a thin region at the biofilm outer edge, the agreement is good; the errors are generally less than $20 \%$. This suggests that each cell expresses only one of the three genes we consider and that the average expression levels are constant throughout the biofilm at these early times. At later times, $t>35 \mathrm{~h}$, the gene expression levels decrease and the agreement worsens.

Our analysis also reveals the spatiotemporal dependence of biofilm composition, whereas the previous flow cytometry study (Vlamakis et al. 2008) only provides the composition of the whole biofilm at discrete times. In the outer region, close to the biofilm edge, the majority component changes from motile to matrix at $25 \mathrm{~h}$ and, at $40 \mathrm{~h}$, switches to either low-fluorescing or sporulating, as shown in Fig. 7c and Movie $\mathrm{S} 1 \mathrm{~F}$ ). In the inner region, $r<1 \mathrm{~mm}$, the majority component initially is motile, whereas at $50 \mathrm{~h}$, it switches to lowfluorescence material and, after $55 \mathrm{~h}$, the majority component becomes sporulating cells. The effective heights of the three fluorescent cell types are shown in Fig. 7d-f and Movie S1 (B-D), respectively, where the maximum is mirrored in the majority plot in Fig. 7c. We observe that sporulation is detectible about $5 \mathrm{~h}$ after colonization; this is shown in Fig. $7 \mathrm{f}$ by the black dashed line that is temporally behind the colonization front, shown by the continuous black line, with a delay time of $\Delta t=5 \mathrm{~h}$.

We graphically illustrate that biofilm growth is a not a local process and that the growth of a patch of the biofilm not only depends on its local age but also involves several nonlocal inputs. We create kymographs using the colonization time, $\Delta t$, which records the duration that a particular patch has been colonized by the bacteria. Neglecting the slow expansion of the biofilm surface, the colonization time registers the local 
Fig. 7 Kymographs of biofilm growth, where the spatial dimension is the radial direction, $r$, and azimuthal averages are taken. a The total height of the biofilm obtained from the optical density with $\lambda=1.2 \mathrm{~mm}$. b The discrepancy between the height from the optical density and that obtained from the fluorescence using Eq. (3), which we attribute to an increase in the amount of low-fluorescent or nonfluorescent materials. c The majority biofilm component, including the lowfluorescence materials. d-f The height of the motile, matrixproducing, and sporulating cells, respectively. The dashed black line indicates the points in time and space where the substrate has been colonized for $5 \mathrm{~h}$. The radius of the front is indicated by the black curves in $\mathbf{a}, \mathbf{d}, \mathbf{e}$, and $\mathbf{f}$

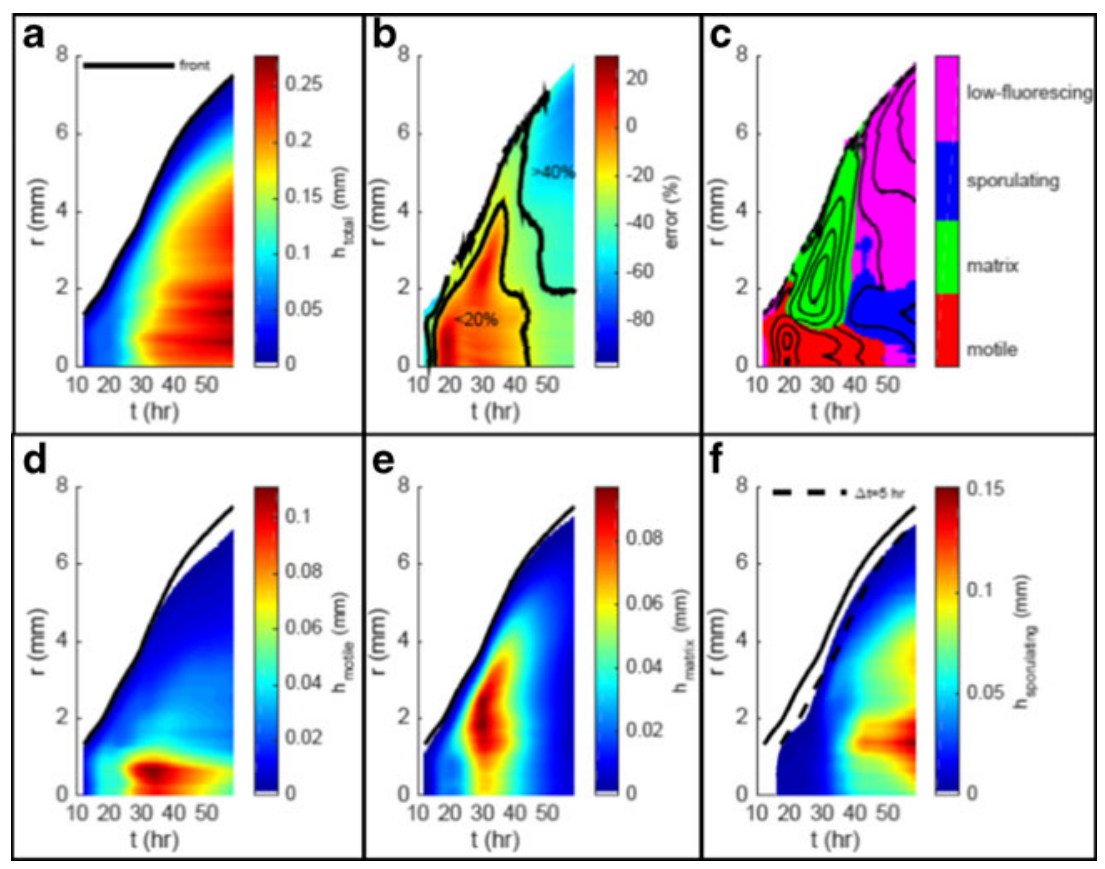

age of each biofilm region from the point in time when it was first colonized. This concept is exemplified by the sporulating phenotype, where at each location, sporulation is first observed $5 \mathrm{~h}$ after colonization: The dashed black line in Fig. $6 \mathrm{f}$ indicates the appearance of sporulation and is translated forward in time by about $5 \mathrm{~h}$ with respect to the colonization front, indicated by the solid line. We further use the colonization time to examine overall biofilm growth, the growth rates represented by $d_{t} h_{i}$ are time derivatives in height $h_{i}$, the unit is $\mathrm{mm} / \mathrm{h}$. The biofilm growth rate $d_{t} h_{\text {total }}$ is greatest shortly after colonization, and it then diminishes, as shown in Fig. 8a. The growth rate is far greater for the range of radial distances $3<\mathrm{r} / \mathrm{mm}<4$, which supports the nonlocal character of biofilm growth. The growth rate of the motile cells $d_{t} h_{\text {motile }}$
Fig. 8 a-d Kymographs of biofilm growth rates (time derivatives) in terms of the local colonization time and radial distance from the biofilm center for the total height, motile, matrix, and sporulating phenotypes, respectively

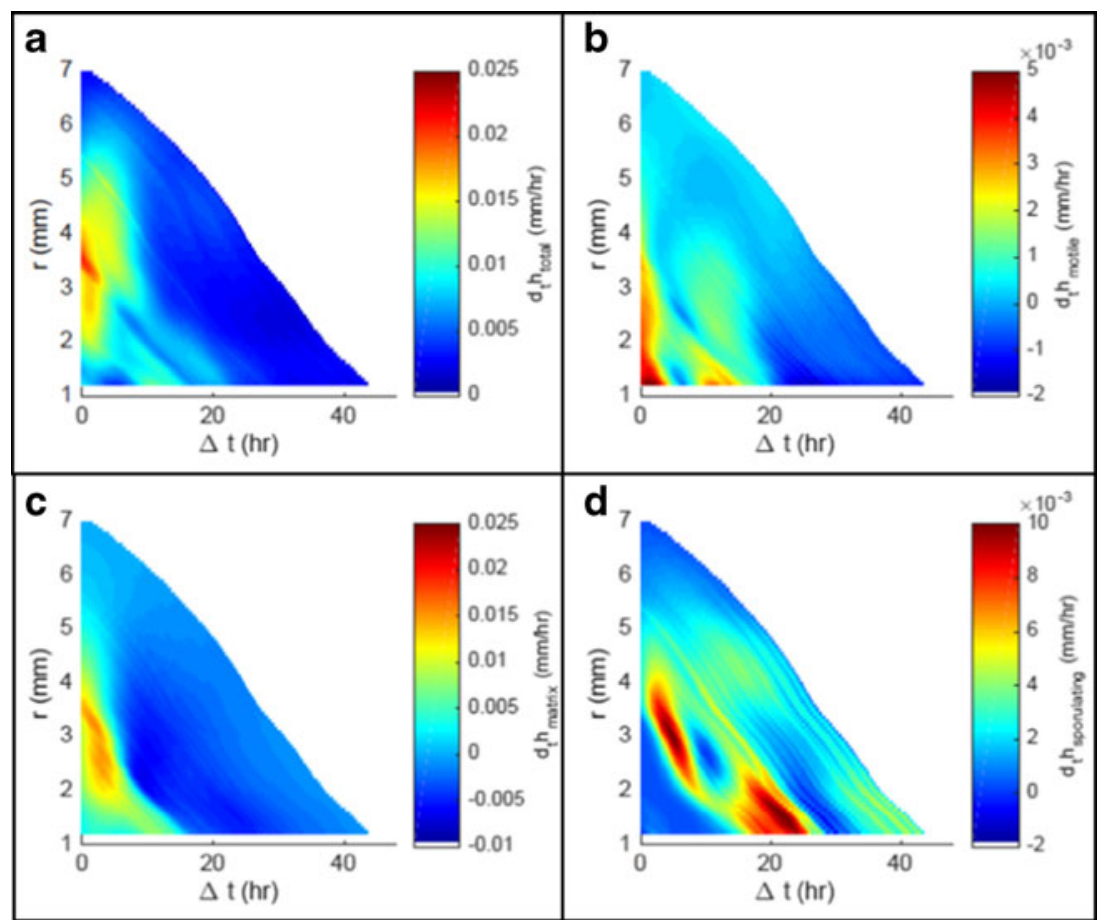


is also greatest at early colonization times and smaller radial distances, $r<3 \mathrm{~mm}$ but is about one order of magnitude smaller than that of the total growth rate, as seen by comparing Fig. $8 \mathrm{~b}$ with Fig. 8a. The growth rate of the matrix-producing cells $d_{t} h_{\text {matrix }}$, shown in Fig. $8 \mathrm{c}$, is close to that of the total biofilm growth rate, indicating that most of the biofilm material is linked to these cells. Finally, the growth rate of the sporulating cells $d_{t} h_{\text {sporulating }}$ is an order of magnitude smaller than the total growth rate, shown in Fig. 8d, and has two local maximal regions again indicating that the growth is nonlocal.

\section{Discussion}

We describe here a technique for quantifying a growing, triple-labeled $B$. subtilis biofilm based on fluorescence and bright-field imaging. Although our analysis relies on several simplifying assumptions and approximations, it possesses the advantage of being noninvasive and providing high-resolution spatiotemporal information. Our technique produced results that are consistent with previous destructive measurements, such as flow cytometry or thin sectioning (Vlamakis et al. 2008), while allowing repeated imaging and tracking of a single biofilm colony over time. We can correlate the physical structures of biofilm colonies (Branda et al. 2004) with the quantity and localization of different cell types in real time. We took advantage of the circular biofilm colony shape to produce spatiotemporal plots of its growth and demonstrated the nonlocal nature of biofilm development by kymographs across the colonization time.

One reason that biofilms are thought to form is to protect the constituent cells from environmental damage and thereby allow the colony to adapt to environmental changes. For instance, infective biofilms are resistant to host defenses and to antibiotic treatment (Hall-Stoodley and Stoodley 2009). Our technique has the potential to allow real-time monitoring of a biofilm as it responds to different stresses, such as biocide treatment, nutrient depletion, or waste accumulation. How do such environmental insults change the cellular differentiation state? What treatment modalities are most effective at inhibiting biofilm growth? In addition to the model species $B$. subtilis, we anticipate that similar techniques may be used for other bacterial species. In addition to its utility as a way to analyze antibiofilm treatments, our method can also be utilized for making comparisons between different growth conditions so as to optimize biofilm growth for specific purposes. For instance, bacteria can be used for soil remediation, soil reinforcement, and environmental waste removal (Vila et al. 2015), and biofilm formation by species such as $B$. subtilis is important for plant protection and growth enhancement (Chen et al. 2013).
Future improvements to our technique will reduce its limitations and try to gain more information by employing additional fluorescent labels. For example, including a fluorescent tag for matrix would make it possible to distinguish between matrix and lowfluorescent and dormant cells. Improving growth rate measurements by sprinkling beads on the surface to measure the local strain field across the surface of the colony, we have already tested the feasibility of this idea with $100-\mu \mathrm{m}$-diameter polystyrene beads; in future experiments, fluorescent beads on unlabeled colonies may yield more accurate results. Stereoimaging is another potentially useful technique; three-dimensional information about the surface morphology would be the most accurate way to find the growth rate. Observation of individual cells in microcolonies may also be a good way to probe phenotypic growth in biofilms; although we studied macroscopic colonies in this work, cell transitions could be directly observed within microcolonies. Existing protocols for growing monolayer colonies on agar pads could be adapted for this technique; this would have the advantage of not requiring correction for the attenuation of light in the biofilm.

This type of fluorescent image analysis can also be extended to other bacterial species or even cell cultures that are appropriately labeled. We are applying our technology to explore other important phenotypes such as competent cells and cannibalistic cells. The two necessary steps are to fluorescently label the relevant phenotypes and to characterize the optical properties of the biofilm. This allows conversion of fluorescence microscope images to subpopulations of each phenotype.

This method could be applied to numerous other biofilm systems. Labeled versions of mutant $B$. subtilis strains could yield further insight into the structure and timing of sporulation. Matrix knockouts do not produce wrinkles, providing an easy way to tell whether channels are allowing nutrients to flow to the centers of the colonies. Strains labeled for surfactin or other intercellular signal molecules could elucidate the dynamics of cellular communication during biofilm development. Labeling surfactin in B. subtilis has not been feasible so far, but analogous labels are available in Pseudomonas.

This method could also be used to suggest new mechanisms for phenotypic switching in multispecies biofilms. We are using this technique to explore cell motion in biofilms; because we do not destroy the biofilm during our observation, we are able to explore bacterial cells movement in different regions of the growing biofilm, such as near the center where there are more motile cells, and also near edge where there are also very motile cells. In addition, by adjusting the agar plate thickness, we can use our technique to explore cell movement underneath the biofilm. This will enable us to explore cell mechanisms of movement during biofilm growth. 
Acknowledgments We thank Richard Losick's group and Roberto Kolter's group for providing triple-fluorescent-labeled Bacillus subtilis strains. This work was supported by the National Science Foundation (DMR-1310266, DMS-1411694), the Harvard Materials Research Science and Engineering Center (DMR-1420570), the National Natural Science Foundation of China (11272002), and the Beijing Higher Education Young Elite Teacher Project (YETP0363).

\section{Compliance with ethical standards}

Funding The National Science Foundation (DMR-1310266, DMS1411694), the Harvard Materials Research Science and Engineering Center (DMR-1420570), the National Natural Science Foundation of China (11272002), and the Beijing Higher Education Young Elite Teacher Project (YETP0363).

Conflict of interest The authors declare that they have no conflict of interest.

Ethical approval This article does not contain any studies with human participants or animals performed by any of the authors.

\section{References}

Barnett TA, Valenzuela D, Riner S, Hageman JH (1983) Production by Bacillis subtilis of brown sporulation-association pigments. Can $\mathrm{J}$ Microbiol 29(1):96-101

Branda SS, González-Pastor JE, Ben-Yehuda S, Losick R, Kolter R (2001) Fruiting body formation by Bacillus subtilis. Proc Natl Acad Sci U S A 98:11621-11626

Branda SS, González-Pastor JE, Dervyn E, Ehrlich SD, Losick R, Kolter $\mathrm{R}$ (2004) Genes involved in formation of structured multicellular communities by Bacillus subtilis. J Bacteriol 186(12):3970-3979

Chang S, Cohen SN (1979) High frequency transformation of Bacillus subtilis protoplasts by plasmid DNA. Mol Gen Genet MGG 168(1): 111-115

Chen Y, Yan F, Chai Y, Liu H, Kolter R, Losick R, Guo JH (2013) Biocontrol of tomato wilt disease by Bacillus subtilis isolates from natural environments depends on conserved genes mediating biofilm formation. Environ Microbiol 15(3):848-864

Chudakov DM, Matz MV, Lukyanov S, Lukyanov KA (2010) Fluorescent Proteins and Their Applications in Imaging Living Cells and Tissues. Physical Rev 90:1103-1163. doi:10.1152/ physrev.00038.2009

Costerton J, Stewart PS, Greenberg E (1999) Bacterial biofilms: a common cause of persistent infections. Science 284(5418):1318-1322

Curtis PD, Taylor RG, Welch RD, Shimkets LJ (2007) Spatial organization of Myxococcus xanthus during fruiting body formation. J Bacteriol 189(24):9126-9130

Dervaux J, Magniez JC, Libchaber A (2013) On growth and form of Bacillus subtilis biofilms. Interface Focus 4(6):20130051

Doherty GP, Bailey K, Lewis PJ (2010) Stage-specific fluorescence intensity of GFP and mCherry during sporulation In Bacillus subtilis. BMC Res Notes 3:303

Dubnau D (1991) Genetic competence in Bacillus subtilis. Microbiol Rev 55:395-424

Dubnau D, Provvedi R (2000) Internalizing DNA Res Microbiol 151: $475-480$

Eisele TC, Gabby KL (2014) Review of Reductive Leaching of Iron by Anaerobic Bacteria. Miner Process Extr Metall Rev 35(2):75-105
Evdokimov AG, Pokross ME, Egorov NS, Zaraisky AG, Yampolsky IV, Merzlyak EM, Shkoporov AN, Sander I, Lukyanov KA, Chudakov DM (2006) Structural basis for the fast maturation of Arthropoda green fluorescent protein. EMBO Rep 7:1006-1012

Falk MM, Baker SM, Gumpert AM, Segretain D, Buckheit RW (2009) Gap junction turnover is achieved by the internalization of small endocytic double-membrane vesicles. Mol Biol Cell 20:3342-3352

Gardel EJ, Nielsen ME, Grisdela PT, Girguis PR (2012) Duty cycling influences current generation in multi-anode environmental microbial fuel cells. Environ Sci Technol 46(9):5222-5229

Golden JW, Yoon HS (2003) Heterocyst development in Anabaena. Curr Opin Microbiol 6(6):557-563

Gonzalez-Pastor JE, Hobbs EC, Losick R (2003) Cannibalism by sporulating bacteria. Science 301:510-513

Hall-Stoodley L, Stoodley P (2009) Evolving concepts in biofilm infections. Cell Microbiol 11(7):1034-1043

Hottes AK, Shapiro L, McAdams HH (2005) DnaA coordinates replication initiation and cell cycle transcription in Caulobacter crescentus. Mol Microbiol 58(5):1340-1353

Kearns DB, Losick R (2005) Cell population heterogeneity during growth of Bacillus subtilis. Genes Dev 19:3083-3094

Kearns DB, Chu F, Branda SS, Kolter R, Losick R (2005) A master regulator for biofilm formation by Bacillus subtilis. Mol Microbiol 55(3):739-749

Logan BE (2008) Microbial fuel cells. John Wiley \& Sons

Mazza F (1994) The use of Bacillus subtilis as an antidiarrhoeal microorganism. Boll Chim Farm 133(1):3-18

O’Toole G, Kaplan HB, Kolter R (2000) Biofilm formation as microbial development. Annu Rev Microbiol 54(1):49-79

Piggot PJ, Hilbert DW (2004) Sporulation of Bacillus subtilis. Curr Opin Microbiol 7:579-586

Rudner DZ, Losick R (2001) Morphological coupling in development: lessons from prokaryotes. Dev Cell 1:733-742

Seminara A, Angelini TE, Wilking JN, Vlamakis H, Ebrahim S, Kolter R, Weitz DA, Brenner MP (2012) Osmotic spreading of Bacillus subtilis biofilms driven by an extracellular matrix. Proc Natl Acad Sci U S A 109(4):1116-1121

Shemesh M, Chai Y (2013) A combination of glycerol and manganese promotes biofilm formation in Bacillus subtilis via the histidine kinase KinD signaling. J Bacteriol 195:2747-2754

Singh R, Paul D, Jain RK (2006) Biofilms: implications in bioremediation. Trends Microbiol 14(9):389-397

Swain MR, Ray RC (2009) Biocontrol and other beneficial activities of Bacillus subtilis isolated from cowdung microflora. Microbiol Res 164(2):121-130

Verplaetse E, Slamti L, Gohar M, Lereclus D (2015) Cell Differentiation in a Bacillus thuringiensis population during planktonic growth, biofilm formation, and host infection. Mbio 6(3):00138-00115

Vila J, Tauler M, Grifoll M (2015) Bacterial PAH degradation in marine and terrestrial habitats. Curr Opin Biotechnol 33:95-102

Vlamakis H, Aguilar C, Losick R, Kolter R (2008) Control of cell fate by the formation of an architecturally complex bacterial community. Genes Dev 22:945-953

Vlamakis H, Chai YR, Beauregard P, Losick R, Kolter R (2013) Sticking together: building a biofilm the Bacillus subtilis way. Nat Rev Microbiol 11(3):157-168

Wiedenmann J, Oswald F, Nienhaus GU (2009) Fluorescent proteins for live cell imaging: opportunities, limitations and challenges. IUBMB Life 61(11):1029-1042 